\title{
Simulierter zeitlicher Schneedeckenverlauf und Schneedeckendauer des Oberen Pinzgau berechnet auf Basis eines mehrfach validierten hydrologischen Modells
}

Simulated snow-cover time curve and snow-cover duration in the Upper Pinzgau valley (River Salzach in the Province of Salzburg) based on a hydrological model validated on the basis of several data sets von G. Koboltschnig ${ }^{1}$, W. Schöner ${ }^{2}$ und H. Holzmann ${ }^{3}$

\section{Kurzfassung/Summary}

Das Einzugsgebiet der Oberen Salzach bis zum Pegel Mittersill wurde mit dem hydrologischen Modell PREVAH untersucht. In einer vorangegangenen Studie wurde das Modell ausgehend von stündlichen Abflussbeobachtungen, Fernerkundungsdaten der SchneeverteilungundmitHilfevon beobachteten Gletschermassenbilanzdaten mehrfach validiert. Das Spektrum der Modellergebnisse erstreckt sich von den einzelnen zum Abfluss beitragenden Komponenten wie dem Direktabfluss aus flüssigem Niederschlag, dem Schnee- und Gletscherschmelzabfluss und dem Abfluss aus den Bodenspeichern, über Gletschermassenbilanzen bis hin $\mathrm{zu}$ den flächenverteilten Informationen des Schneedeckenzustandes. Verglichen mit aus Punktmessdaten angepassten Verteilungen der mittleren Schneedeckendauer für gewisse Zeitperioden besteht durch diese Methode der entscheidende Vorteil für einzelne Jahre und durch die Wasserbilanz validierte Darstellungen der Schneedeckendauer zu generieren. Die Depletion-Curves zeigen für die berechneten Haushaltsjahre recht unterschiedliches Verhalten, welche die Witterung des Haushaltsjahres widerspiegeln. Die Anzahl der Tage mit 100\%iger Schneebedeckung schwankt zwischen 7 Tagen für 2002/2003 und 107 Tagen für das Haushaltsjahr 1999/2000. Die mittlere Schneedeckendauer wurde für den Modellierungszeitraum (Oktober 1999 bis September 2005) mit 230 Tagen berechnet, was einen um
14 Tage kürzeren Mittelwert im Vergleich zu den aus Stationsmessungen berechneten Daten für die Periode 1961 - 1990 ergibt.

The catchment area of the Upper Salzach above the Mittersill riverflow gauge has been studied using the hydrological model PREVAH. The model was multi-validated in a previous study, on the basis of hourly flow recordings, remotesensing data of snow distribution and using glacier mass-balance records. The results obtained from the model include the various components making up flow, such as direct runoff from rainfall, runoff from snow and glacier melt and flow from groundwater reservoirs, as well as glacier mass balances and information on the spatial distributed snow cover condition. Compared with adjusted mean snow-cover duration distributions, generated from point measurements, for certain periods in time, this method offers the great advantage of permitting the generation of snow-cover duration curves, validated on the basis of water balance, and this for individual years. The depletion curves exhibit a fairly varying behaviour for the years when the balance was studied, thus reflecting variations in weather conditions. The number of days of $100 \%$ snow cover varied between 7 days for 2002-03 and 107 days for the balance year 19992000. The mean snow-cover duration has been calculated to be 230 days for the study period (October 1999 to September 2005), which is 14 days shorter than the mean value calculated from station records for the period 1961 to 1990.

\section{Einleitung}

Im Zuge des Projektes SNOWTRANS (gefördert durch die Österreichische Akademie der Wissenschaften im Programm HOE 29) wurde das Einzugsgebiet der Oberen Salzach bis zum Pegel Mittersill als Modellgebiet für die Modellierung mit dem Wasserbilanzmodell PREVAH (Gurtz et al., 1999) ausgewählt. Dabei war das Ziel, den Abfluss und vor allem die Gletscher- und Schneeschmelze als wesentliche zum Abfluss beitragende Komponenten auf stündlicher Basis numerisch zu simulieren. Das hydrologische Modell wurde nicht ausschließlich mit den beobachteten Abflussdaten am Gebietsauslass, sondern auch unter zu Hilfenahme von Satellitenfernerkundungsdaten der Schneebedeckungsmuster und mit beobachteten Gletschermassenbilanzdaten mehrfach validiert (Koboltschnig et al., 2008a). Aus klimatologischer Sicht wurden für den Alpenraum (Beniston, 1997; Wielke et al., 2004) und für Österreich speziell (Hantel et al., 2000) bereits Untersuchungen zur Schneedeckendauer und deren Abhängigkeit von der Lufttemperatur durchgeführt. Die Frage nach bisherigen Trends und zukünftigen Änderungen bekommt in den Fachbereichen der Bioklimatologie (Randin et al., 2008) aber auch in der Hydrologie (Strasser und Mauser, 2001) zunehmend größere Bedeutung. In der Hydrologie ist dabei die Dauer der Speicherung von Wasser in der Schneedecke und die Verfügbarkeit und Interaktion von flüssigem Niederschlag, welcher auf die Schneedecke fällt 
von Bedeutung (siehe z. B. Singh et al., 1997), da dadurch die Abflussbildung vor allem bei Hochwasser maßgeblich beeinflusst werden kann.

\section{Das Untersuchungsgebiet}

Das hydrologische Einzugsgebiet des Oberen Pinzgau bis zum Pegel Mittersill hat ein Fläche von $593 \mathrm{~km}^{2}$ und ist durch die Kombination von vergletscherten Flächen, hochalpinen, vegetationsfreien Gebieten aber auch durch bewaldete Zonen und landwirtschaftlich genutzte Flächen im Talboden geprägt. Die Höhenverteilung im Einzugsgebiet erstreckt sich von $780 \mathrm{~m}$ ü.d.A. beim Pegel Mittersill bis auf $3666 \mathrm{~m}$ ü.d.A. auf den Großvenediger. Alle hydrometeorologischen Beobachtungsdaten wurden vom Hydrographischen Dienst Salzburg (HD), der ZAMG (Zentralanstalt für Meteorologie und Geodynamik), der AHP (Austrian Hydro Power) und vom Lawinenwarndienst Salzburg und Tirol (LWD) bezogen. Eigene Messungen wurden für diese Studie nicht durchgeführt.

Das Einzugsgebiet umfasst die nördliche Abdachung des Venedigergebietes mit den großen SüdNord erstreckten Zubringern aus den Tauerntälern: Krimmlerache, Obersulzbach, Untersulzbach, Habach und Hollersbach (von Westen nach Osten). Weiters kommen noch die nördlichen Zubringer aus den unvergletscherten Kitzbüheler Alpen dazu. Die vergletscherte Fläche des Einzugsgebietes, ermittelt aus dem CORINE Landnutzungsdatensatz aus dem Jahr 2000 beträgt $37.3 \mathrm{~km}^{2}$. Die Klassifizierung von CORINE wird jedoch für Gletscher als „Schnee und Gletscher" angegeben. Für die Skala dieser Untersuchung ist diese Unterteilung ungeeignet. Das Orthofoto einer Befliegung im Jahr 2003 (ASL, 2003) wurde daher für die Digitalisierung der Flächen herangezogen, und es konnte eine Fläche von

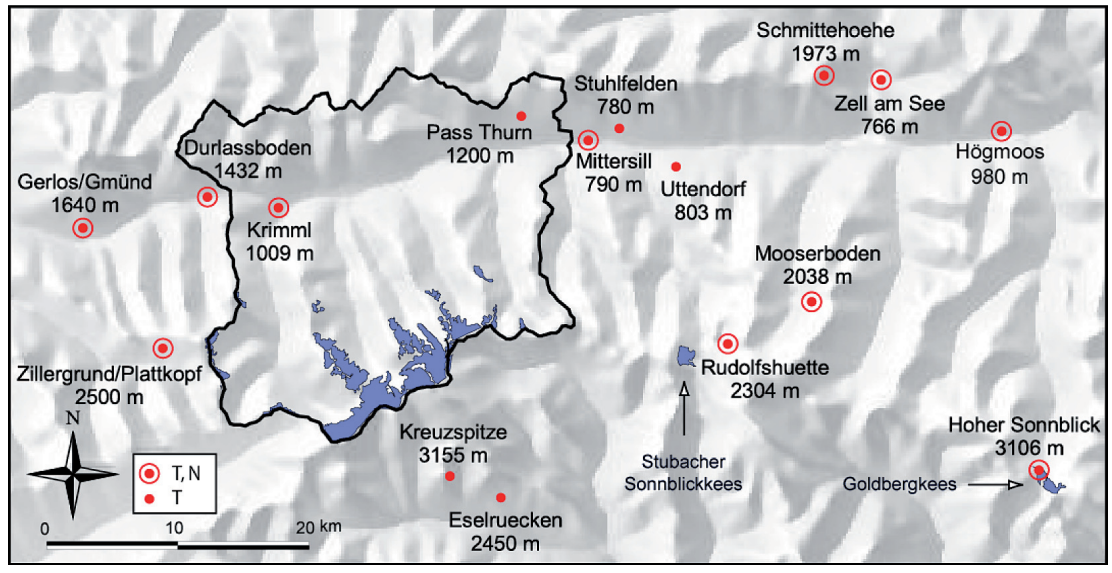

Abb. 1: Das Einzugsgebiet des Oberen Pinzgau und meteorologische Stationen mit stündlicher Auflösung (rote Punkte = nur Temperaturmessungen, roter Punkt mit Kreis = zumindest Temperatur und Niederschlagsmessung). Blau dargestellt sind die Gletscherflächen.

\section{Tab. 1: Flächenanteile der Höhenzonen im Einzugsgebiet Oberer} Pinzgau

\begin{tabular}{ccc}
\hline $\begin{array}{c}\text { Höhenzone } \\
\text { [m ü.d.A] }\end{array}$ & $\begin{array}{c}\text { Fläche } \\
{\left[\mathbf{k m}^{2}\right]}\end{array}$ & $\begin{array}{c}\text { Anteil an der } \\
\text { Gesamtfläche [\%] }\end{array}$ \\
\hline $700-1000$ & 46 & 8 \\
\hline $1000-1500$ & 105 & 17 \\
\hline $1500-2000$ & 170 & 29 \\
\hline $2000-2500$ & 164 & 28 \\
\hline $2500-3000$ & 97 & 16 \\
\hline $3000-3700$ & 10 & 2 \\
\hline$\Sigma$ & 593 & 100 \\
\hline
\end{tabular}

$32.8 \mathrm{~km}^{2}$ ermittelt werden. Das entspricht einem gletscherbedeckten Anteil von $5.3 \%$.

Die mittlere Höhe des Einzugsgebietes Oberer Pinzgau liegt bei etwa $1910 \mathrm{~m}$ ü.d.A. (siehe auch Tab. 1). Die Klassifizierung wurde auf Basis eines DHM mit 10 m Auflösung ermittelt (ASL, 2003). Dabei nehmen die Zonen „1500-2000“ und „2000-2500“ jeweils fast $30 \%$ der Fläche des gesamten Gebietes ein. Der hochalpine Anteil des Einzugsgebietes, also die Zonen von 2500 bis $3700 \mathrm{~m}$, haben einen Anteil von $18 \%$ an der Gesamtfläche. Nur $8 \%$ der Gesamtfläche liegen unter einer Seehöhe von $1000 \mathrm{~m}$ ü.d.A. Der mittlere Jahresniederschlag für das Gebiet bis zum Pegel Mittersill wird für die Klimanormalperiode 1961 - 90 aus der Wasserbilanz mit $1741 \mathrm{~mm}$, die Wasserbilanzkomponenten „Aktuelle Verdunstung“ mit $311 \mathrm{~mm}$ und die „Mittlere Abflusshöhe" mit $1429 \mathrm{~mm}$ angegeben (Kling et al., 2005). Die Gletscherschmelze wurde dabei nicht berücksichtigt. Für die Beschreibung der weiteren Klimaelemente werden die Analysen der langjährigen Beobachtungen der Klimastation Zell am See (766 m ü.d.A.) herangezogen (Auer et al., 2001). Dabei wird das Klima als inneralpin mit starker Tagesamplitude der Lufttemperatur, sonnig im Winter und im Sommer oft Quellbewölkung vom Alpenhauptkamm her beschrieben. Die Lufttemperatur beträgt im Mittel (1961-90) 6,9 ${ }^{\circ} \mathrm{C}$ mit etwa 130 Frosttagen, 41 Eistagen und 32 Sommertagen. Frosttage sind jene Tage, an welchen das Minimum der Lufttemperatur unter $0^{\circ} \mathrm{C}$ liegt. Ist an solchen Tagen auch das Maximum der Lufttemperatur (die Tageshöchsttem- 
peratur) unter $0^{\circ} \mathrm{C}$, so spricht man von Eistagen. Tage, an welchen die Lufttemperatur $25^{\circ} \mathrm{C}$ erreicht oder überschreitet, werden als Sommertage bezeichnet. Der inneralpine Charakter setzt sich im Einzugsgebiet des Oberen Pinzgau fort. Durch die größere Höhenlage gibt es entsprechend einen höheren Anteil an Frost- und Eistagen.

\section{Methodik}

\subsection{Hydrologisches Modell PREVAH}

PREVAH (Precipitation-RunoffEvapotranspiration-Hydrotope Model; Gurtz et al., 1999) ist ein HRU (Hydrological Response Unit) basiertes Modell, das die hydrologische Antwort der einzelnen HRUs, in welche das Einzugsgebiet aufgeteilt wird auf den meteorologischen Input berechnet. Für die in dieser Arbeit vorgestellten Modellrechnungen wurde ein erweitertes Tag-Grad-Verfahren mit potentieller Solarstrahlung verwendet (Hock, 1999). Dieses Schmelzmodell benötigt ausschließlich die gemessene Lufttemperatur als Input und berechnet für jedes Flächenelement die potentielle Solarstrahlung, welche für die notwendige Ausprägung des Tagesganges bei den durch Gletscher- und Schneeschmelze beeinflussten Abflüssen sorgt. Eine detaillierte Beschreibung für das Modell liefert Viviroli et al. (2007).

\subsection{Hydro- meteorologische Datenbasis für das Ein- zugsgebiet Oberer Pinzgau}

Für die Kalibrierung bzw. Validierung des hydrologischen Modells waren diehydro- meteorologischen Daten für den Zeitraum Oktober 1999 bis Oktober 2005 verfügbar. Tab. 2 gibt einen Überblick der im und nahe dem Einzugsgebiet gelegenen meteorologische Stationen auf stündlicher Basis. Die höchst-

Tab. 2: Stationen mit stündlichen meteorologischen Aufzeichnungen

\begin{tabular}{|c|c|c|c|}
\hline Stationsname & Betreiber & Seehöhe [m] & Messgröße \\
\hline Krimml & ZAMG & 1009 & $\mathrm{~T}, \mathrm{~N}, \mathrm{~S}, \mathrm{Vw}$ \\
\hline Rudolfshütte & ZAMG & 2304 & $\mathrm{~T}, \mathrm{~N}, \mathrm{~S}, \mathrm{Vw}, \mathrm{G}, \mathrm{Rf}$ \\
\hline Hoher Sonnblick & ZAMG & 3106 & $\mathrm{~T}, \mathrm{~N}, \mathrm{~S}, \mathrm{Vw}, \mathrm{G}, \mathrm{Rf}$ \\
\hline Uttendorf & ZAMG & 803 & $\mathrm{~T}$ \\
\hline Schmittenhöhe & ZAMG & 1973 & $\mathrm{~T}, \mathrm{~N}, \mathrm{~S}, \mathrm{Vw}, \mathrm{G}, \mathrm{Rf}$ \\
\hline Zell am See & ZAMG & 766 & $\mathrm{~T}, \mathrm{~N}, \mathrm{~S}, \mathrm{Vw}$ \\
\hline Pass Thurn & $\mathrm{HD}$ & 1200 & $\mathrm{~T}$ \\
\hline Stuhlfelden & HD & 780 & $\mathrm{~T}$ \\
\hline Mittersill & $\mathrm{HD}$ & 790 & $\mathrm{~N}$ \\
\hline Eselrücken & LWD & 2450 & $\mathrm{~T}, \mathrm{Rf}$ \\
\hline Kreuzspitze & LWD & 3155 & T, Rf, Vw \\
\hline Durlaßboden & $\mathrm{AHP}$ & 1432 & T, N \\
\hline Högmoos & $\mathrm{AHP}$ & 980 & T, N \\
\hline Mooserboden & AHP & 2038 & T, N \\
\hline Gerlos/Gmünd & AHP & 1640 & T, N \\
\hline Schwarzach & AHP & 600 & $\mathrm{~T}, \mathrm{~N}$ \\
\hline Zillergrund/Plattkopf & AHP & 2500 & $\mathrm{~T}, \mathrm{~N}$ \\
\hline
\end{tabular}

gelegenen Stationen sind „Sonnblick“ und „Kreuzspitze“. Anzumerken ist, dass auf Grund der Wartung und der Fehlerprüfung die größte Qualität der Messdaten von den Stationen der ZAMG zu erwarten ist. Die räumliche Verteilung der meteorologischen Stationen ist in Abb. 1 dargestellt. Nur die zwei Stationen „Krimml“ und „Paß Thurn" befinden sich innerhalb des Einzugsgebietes. Strahlungsmessungen (ausschließlich für die Verdunstungsberechnung) konnten nur von Stationen der ZAMG verwendet werden. Die Verteilung der Stationen in den Höhenzonen scheint ausreichend abgedeckt $\mathrm{zu}$ sein.

\subsection{Modelltechnische Untertei- lung des Einzugsgebietes}

Als Basis für die Simulation stand ein hochauflösendes Höhenmodell $(10 \times 10 \mathrm{~m})$ zur Verfügung. Im „Preprocessing“ wurde das Höhenmodell durch ein „Resampling“ auf eine gröbere Auflösung von $150 \times 150$ m gebracht. Damit wird das Gebiet von einem $214 \times 203$ Raster abgedeckt. Das entspricht einer Zellenanzahl von 26434 innerhalb der Einzugsgebietsgrenzen. Die Unterteilung in HRUs mit dem „Preprocessor“ von PREVAH (Gurtz et al., 1999) wurde nach folgenden Kriterien durchgeführt:

- Subeinzugsgebiete (Gewässer dritter Ordnung) $=5$ Klassen

- Höhenzonen $(100 \mathrm{~m})=28$ Klassen

- Exposition (Haupt-, Zwischenhimmelsrichtungen + flach $)=$ 9 Klassen

- Landbedeckung = 10 Klassen

- Gletscher (Firn und Eis, Gleichgewichtslinie bei $2900 \mathrm{~m}$ ) = 2 Klassen

Daraus ergeben sich 1571 HRUs und 109 meteorologische Zonen. Für die meteorologischen Zonen wurden nur die ersten beiden Kriterien der HRU Unterteilung an- 
genommen. Die Gletscherflächen entsprechen der Digitalisierung der Befliegungsauswertung von 2003. Die Unterteilung in HRUs entspricht einer Reduktion der zu berechnenden Teilflächen um den Faktor 17 gegenüber einem vollverteilten Rastermodell (Koboltschnig et al., 2008a). Die flächenhaften Ergebnisse der Modellierung werden als Raster ausgegeben, wobei die HRU's auf Raster abgebildet werden.

\subsection{Meteorologischer Input}

Durch Interpolation der verfügbaren Stationsdaten wurde der Modellinput generiert. Zur Anwendung kam für alle meteorologischen Größen, ausgenommen der Lufttemperatur, das IDW (Inverse Distance Weighting)-Verfahren. Die Lufttemperaturdaten wurden mit dem „IDW-Lapsrate“Verfahren regionalisiert, wobei die Stationsdaten mit dem vorgegebenen Höhengradienten auf ein einheitliches Höhenniveau gebracht und auf diesem mittels IDW interpoliert werden. Anschließend wird mit Hilfe des Höhengradienten das interpolierte Raster mit den Daten des Höhenmodells wieder auf die Geländehöhe gebracht. Der Temperaturgradient wurde mit $0.6^{\circ} \mathrm{C} / 100 \mathrm{~m}$ angesetzt - dieser Wert ergibt sich aus langjährigen Temperaturmittelwerten. Mitunter wird der Gradient zwischen zwei Stationen wie z.B. Rudolfshütte und Uttendorf noch kleiner (etwa $\left.0.5^{\circ} \mathrm{C} / 100 \mathrm{~m}\right)$. Das ist durch die Ausbildung von Kaltluftseen und der stabilen Inversion im Winter erklärbar.

\subsection{Modellkalibrierung}

Als Kalibrierungsperiode wurde ein Zeitraum von drei Jahren, vom 01.10.1999 bis zum 30.09.2002 gewählt. Die Modellparameter in PREVAH wurden mit einer automatischen Optimierungsroutine anhand der beobachteten stündlichen Abflussdaten vom Pegel Mittersill (siehe Abb.1) kalibriert. Die Kalibrierung erfolgt durch Optimierung einer objektiven Zielfunktion (Zappa und Kan, 2007), bei der die lineare Modellgüte (Nash \& Sutcliffe, 1970) der gesamten Kalibrierungsperiode optimiert wird. Dabei wurde der in Gurtz et al. (1999) vorgeschlagene Satz von elf Parameterpaaren verwendet. Ein weiteres Feintuning der Parameter wurde anschließend noch „von Hand“ durchgeführt. Dabei wurden die Speicherparameter für Eis, Firn und Schnee, die in der automatischen Kalibrierung nicht implementiert sind, angepasst. Dabei konnten die Parameter aus vorangegangenen Untersuchungen am Goldbergkees im Gebiet des Hohen Sonnblicks übertragen werden (Koboltschnig et al., 2007). Die Korrektur des festen Niederschlagsanteils wurde durch die automatisierte Parameteroptimierung mit $44 \%$ ermittelt und im Prozess der händischen Modell-Feinkalibrierung mit Hilfe von Satellitenfernerkundungsdaten auf Grund des beobachteten Ausaperungszustandes des Einzugsgebietes auf $50 \%$ erhöht. Der Niederschlag wurde, getrennt nach festem und flüssigem Anteil separat korrigiert. Durch eine $50 \%$ ige Korrektur des festen Anteils wird der Niederschlagsverteilung ein zusätzlicher Höhengradient „verpasst“. Das kommt durch die Temperaturverteilung in dem stark höhengegliederten Einzugsgebiet zustande, wodurch hoch gelegene Gebiete immer geringere Lufttemperaturen aufweisen und damit der Niederschlag stärker nach oben korrigiert wird.

\subsection{Ermittlung des zeitlichen Verlaufs der Schneebe- deckung}

Durch Auswertung der in Kartenform von PREVAH auf Tagesbasis generierten Speicherzustän- de des Schneewasseräquivalents (SWE) kann der zeitliche Verlauf der Schneebedeckung des Einzugsgebietes berechnet werden. Dazu werden die in Rasterform vorliegenden SWE - Speicherzustände jedes einzelnen Tages nach den Kriterien „schneebedeckt“ oder „schneefrei“ reklassifiziert. Als Grenzwert für das Kriterium „schneebedeckt" wurde ein Schneewasseräquivalent von $1 \mathrm{~mm}$ gewählt. Das entspricht etwa einer Schneehöhe von $1 \mathrm{~cm}$ Neuschnee. Aus der Reklassifizierung lässt sich für jeden modellierten Tag der prozentuelle Anteil der schneebedeckten Fläche der gesamten Einzugsgebietsfläche errechnen.

\subsection{Berechnung der kumulativen Schneedeckendauer}

Ein weiteres Produkt der Schneesimulation in PREVAH ist die Berechnung der kumulativen Schneedeckendauer. Dabei kann vom Modell für jeden Tageszeitschritt ein räumliches Raster generiert werden, welches für jede Rasterzelle die kumulative Schneedeckendauer angibt. Die kumulative Schneedeckendauer ist die Anzahl jener Tage in einem Jahr, an welchen eine Rasterzelle mit zumindest $1 \mathrm{~mm}$ Schneewasseräquivalent gefüllt ist. Aus den Simulationsergebnissen ist damit das für den letzten Tag eines Haushaltsjahres berechnete Raster maßgeblich. Um nun für ein bestimmtes Haushaltsjahr die Schneedeckendauer zu berechnen, wird ein Raster mit der kumulativen Schneedeckendauer am Beginn des Haushaltsjahres von jenem am Ende subtrahiert. Somit ist gewährleistet, dass die maximale Dauer 365 Tage entspricht. Das wurde für alle sechs Haushaltsjahre von 1999 bis 2005 auf dem vorgegebenen Raster von $150 \times 150 \mathrm{~m}$ durchgeführt. Zum Vergleich wurde auch die mittlere Schneedeckendauer der Referenzperiode 1961-1990 gegenübergestellt (250x250m Raster). Diese 
flächenhafte Information ist das Ergebnis einer Interpolation mittels linearer Regression der Schneedeckendauer mit der Seehöhe anhand von Stationsdaten (Schöner \&. Mohnl, 2003). Dabei muss angemerkt werden, dass sich nur zwei Stationen direkt im Einzugsgebiet Oberer Pinzgau befanden.

\section{Ergebnisse}

Das Haushaltsjahr 1999/2000 ist mit 107 Tagen vollständiger Schneebedeckung der Spitzenreiter und 2002/2003 mit sechs Tagen das Schlusslicht (siehe Tab.3). Im Mittel gibt es für den gesamten Simulationszeitraum 1999 bis 2005 66 Tage mit $100 \%$ iger Schneebedeckung.

In Abb. 2 sind die zeitlichen Verläufe für die Jahre 1999 bis 2005 dargestellt. Diese zeigen recht unterschiedliches Verhalten. Einerseits die unterschiedliche Länge der Perioden mit vollständiger Schneebedeckung und andererseits die unterschiedlichen Beginnphasen der Akkumulationsperioden. Die rasch beginnenden und anhaltenden Winterdecken sind in dieser Untersuchungsperiode eher die Minderzahl. Auch in der Ablationsperiode gibt es größere Unterschiede, wobei 2002/2003 als das Haushaltsjahr mit den bisher negativsten beobachtetem Gletschermassenbilanzen (Frauenfelder et al., 2005; Hynek und Schöner, 2004; Koboltschnig et al., 2008b) erwartungsgemäß auch bei der Schneeschmelze den steilsten Abfall der prozentuellen Schneebedeckung zeigt. Der Prozess der Ausaperung der Gletscher ist verglichen mit den anderen Jahren am schnellsten vor sich gegangen und hat damit auch zu den negativen Gletschermassenbilanzen geführt. Bei den minimalen Schneebedeckungen des Einzugsgebietes zeigt auch das Haushaltsjahr 2002/2003 die kleinsten Werte. Dagegen verläuft die Ausaperung im Haushalts-
Tab. 3: Anzahl der Tage mit vollständiger Schneebedeckung des Einzugsgebietes Oberer Pinzgau für den Zeitraum 1999-2005

\begin{tabular}{cc|}
\hline Haushaltsjahr & Anz. d. Tage mit $\mathbf{1 0 0} \%$ Schneebedeckung \\
\hline $1999 / 2000$ & 107 \\
\hline $2000 / 2001$ & 24 \\
\hline $2001 / 2002$ & 75 \\
\hline $2002 / 2003$ & 6 \\
\hline $2003 / 2004$ & 99 \\
\hline $2004 / 2005$ & 99 \\
\hline
\end{tabular}

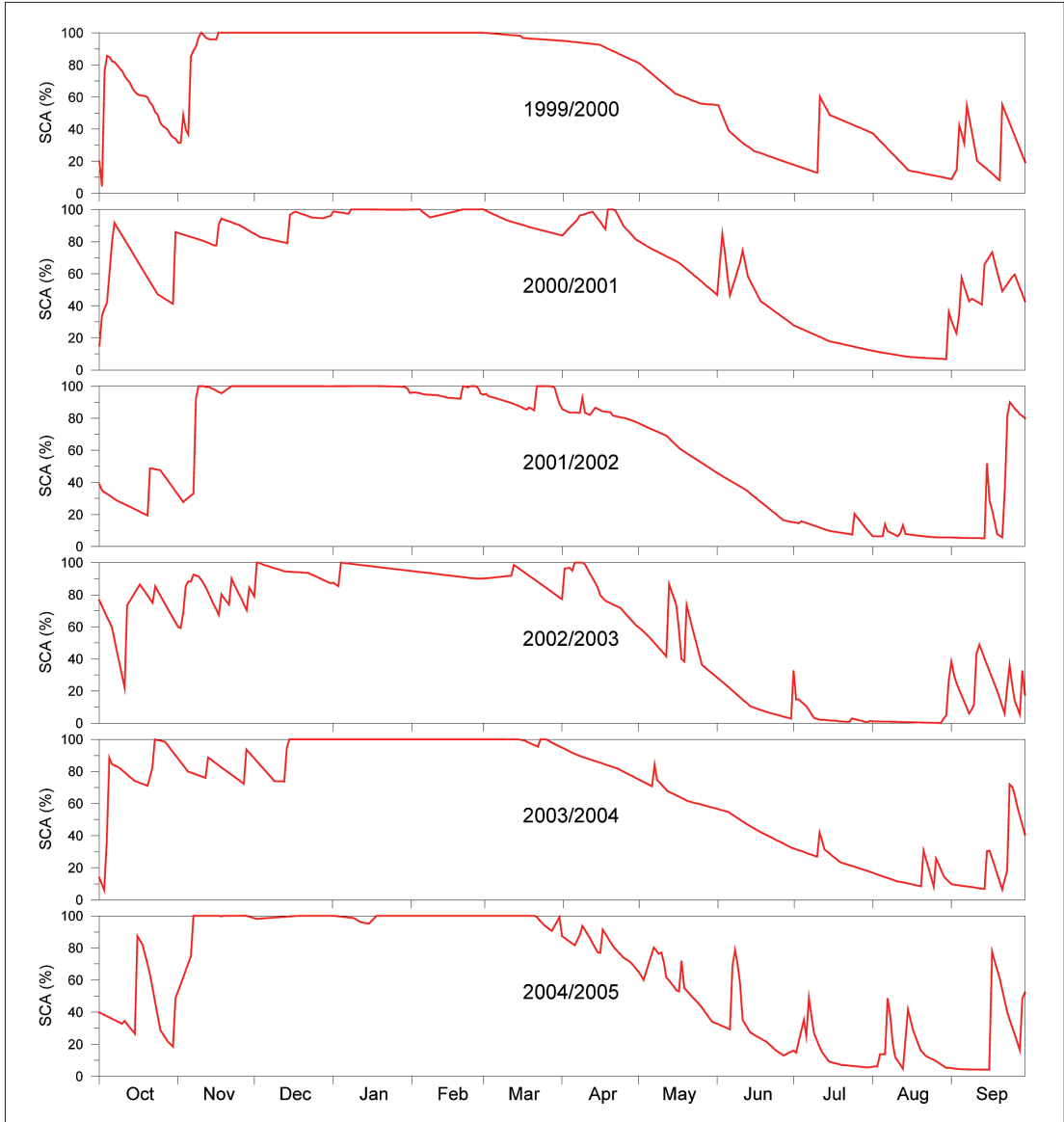

Abb. 2: Zeitlicher Verlauf der prozentuellen Schneebedeckung („Depletion curves“) des Einzugsgebietes Oberer Pinzgau auf Basis der simulierten Tageswerte der Schneebedeckung für die hydrologischen Jahre 1999 bis 2005

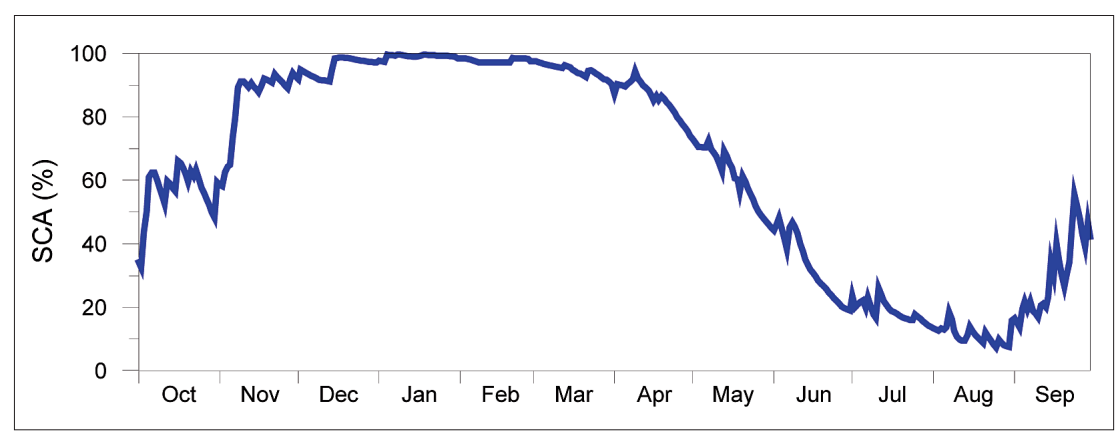

Abb. 3: Mittlere prozentuelle Schneebedeckung der hydrologischen Jahre 1999 bis 2005 des Einzugsgebietes Oberer Pinzgau auf Basis simulierter Tageswerte. 
jahr 2003/2004 deutlich langsamer, es zeigt sich eine flacher geneigte Gerade. Die Unterbrechungen in den Sommermonaten mit kurzzeitig großflächigeren Schneebedeckungen (im Juli 2000 mit $60 \%$ und im August 2005 mit $50 \%$ ) kommen selten vor, nur in der Periode 2004/2005 tritt das häufiger auf. Ab spätestens Mitte September war in allen Haushaltsjahren mit größeren Schneefällen zu rechnen und im Oktober mit dem Beginn des Aufbaus der Winterschneedecke.

Da die zeitlichen Verläufe der relativen Schneebedeckung des Untersuchungsgebietes auch immer wiederkehrende Eigenschaften zeigen, ist es interessant einen mittleren Verlauf der relativen Schneebedeckung darzustellen (Abb. 3). Dabei werden die einzelnen Ausreißer, welche sich durch spontane Schneedeckenzuwächse ergeben, herausgemittelt. Abb. 3 zeigt damit im Mittel sechs Monate im Jahr, in welchen es mehr als $60 \%$ Schneebedeckung im Oberen Pinzgau gab. Ab Ende April beginnt die Schneeschmelzperiode, welche bis Ende Juni gleichbleibend und im Juli und August etwas schwächer verläuft. Im September und Oktober gibt es wieder Neuschneeakkumulation.

\subsection{Kumulative Schneedecken- dauer}

Die angeführten Ergebnisse ergeben große Unterschiede von Jahr zu Jahr. Tab. 4 zeigt, dass 1999/2000 mit 245 Tagen als Gebietsmittelwert als einziges Haushaltsjahr das Mittel der Klimanormalperiode übertrifft.

In Abb. 4 ist das Haushaltsjahr 1999/2000 mit einer langen Schneedeckendauer im Tal und in den großen Höhenzonen zu sehen. 2000/2001 hat eine kürzere Schneedeckendauer im Tal aber eine vergleichbare Dauer wie $1999 / 2000$ in den höheren Lagen. 2001/2002 zeigt wiederum

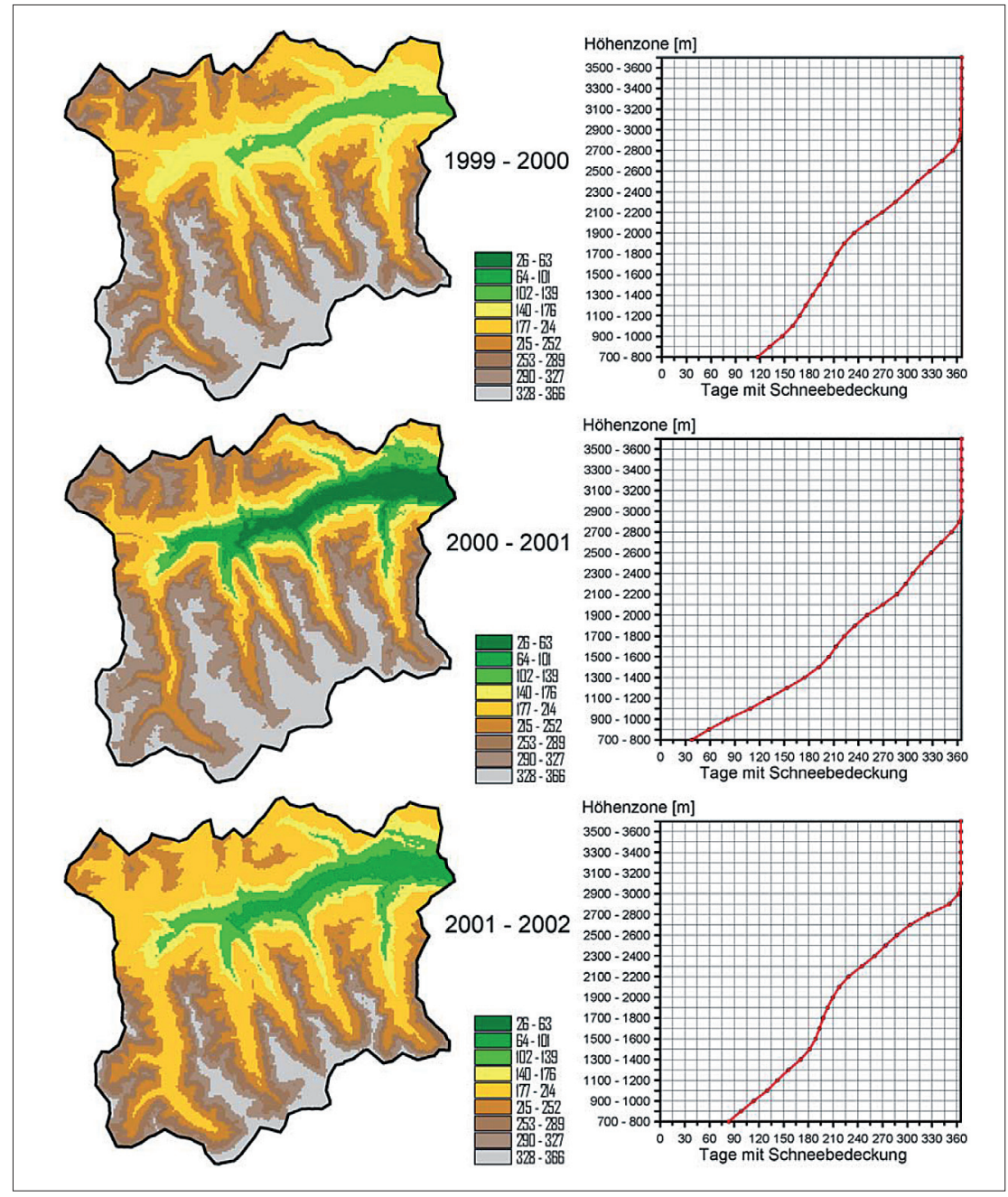

Abb. 4: Räumliche Darstellung der kumulativen Schneedeckendauer der Haushaltsjahre 1999 bis 2002 und mittlere Anzahl der Tage mit Schneebedeckung in den $100 \mathrm{~m}$ - Höhenzonen des Einzugsgebietes Oberer Pinzgau.

Tab. 4: Kennwerte der Schneedeckendauer für die simulierten Haushaltsjahre und die mittleren Perioden 1999-2005 und 1961-1990 im Vergleich

\begin{tabular}{cccc} 
Haushaltsjahr & $\begin{array}{c}\text { Minimum } \\
\text { [Tage] }\end{array}$ & $\begin{array}{c}\text { Maximum } \\
\text { [Tage] }\end{array}$ & Mittel [Tage] \\
\hline $1999 / 2000$ & 105 & 366 & 245 \\
\hline $2000 / 2001$ & 26 & 365 & 241 \\
\hline $2001 / 2002$ & 77 & 365 & 218 \\
\hline $2002 / 2003$ & 58 & 362 & 208 \\
\hline $2003 / 2004$ & 93 & 366 & 244 \\
\hline $2004 / 2005$ & 99 & 365 & 222 \\
\hline Mittel 1999-2005 & 78 & 364 & 230 \\
\hline Mittel 1961-1990 & 105 & 349 & 244 \\
\hline
\end{tabular}

in allen Höhenzonen eine kürzere Schneedeckendauer. Es ist zu beachten, dass die Höhenzonen unterschiedliche Flächenanteile an der Gesamtfläche des Einzugsgebietes haben (siehe Tab. 1). 
Das Haushaltsjahr 2002/2003 zeigt erwartungsgemäß die kürzeste Schneedeckendauer in allen Höhenlagen (Abb. 2.5). Nur in den größten Höhenzonen (ab $3400 \mathrm{~m}$ ü.d.A.) wurde eine ganzjährige Schneedecke simuliert. 2003/2004 zeigt wiederum eine längere Schneedeckendauer in den hohen Lagen. Im Vergleich zu 2004/2005 ist die Schneedeckendauer im Tal ähnlich, nur in den hohen Lagen zeigt 2004/2005 die Klasse der längsten Schneedeckendauer um eine $100 \mathrm{~m}$ Höhenzone weiter oben. In Abb. 6 wird die simulierte mittlere Schneedeckendauer der Periode 1999-2005 mit der beobachteten mittleren (interpolierten) Schneedeckendauer der Periode 1961-1990 (Schöner und Mohnl, 2003) gegenübergestellt. Einerseits ist ein sehr ähnliches, durch die Höhenverteilung vorgegebenes Bild zu sehen. Andererseits zeigen sich für 1999- 2005 in den Tallagen kürzere Schneedeckendauern und in den hohen Lagen größere $\mathrm{Ge}$ biete mit längeren Schneedeckendauern.

\section{Diskussion}

Die hier vorgestellte Arbeit präsentiert einen Ansatz, mit welchem, die Schneedeckendauer und das Ausaperungsverhalten eines Einzugsgebietes basierend auf ein räumlich verteiltes hydrologisches Modell berechnet werden kann. Im Gegensatz zu aus Fernerkundungsdaten abgeleiteter Information zur Schneedeckendauer (z.B. Brander et al., 2000) und dem Ausaperungsverlauf von Gebieten überwiegen die Vorteile der Modellierung auf Grund der Tatsache, dass die InformationzurSchneedeckenverteilung für jeden simulierten Tag berechnet werden kann. Die Verfügbarkeit von Fernerkundungsdaten, sofern es sich um optische Satellitensensoren handelt, ist aber stark von der Wolkenbedeckung abhängig (Parajka und Blöschl, 2006). Eine weitere

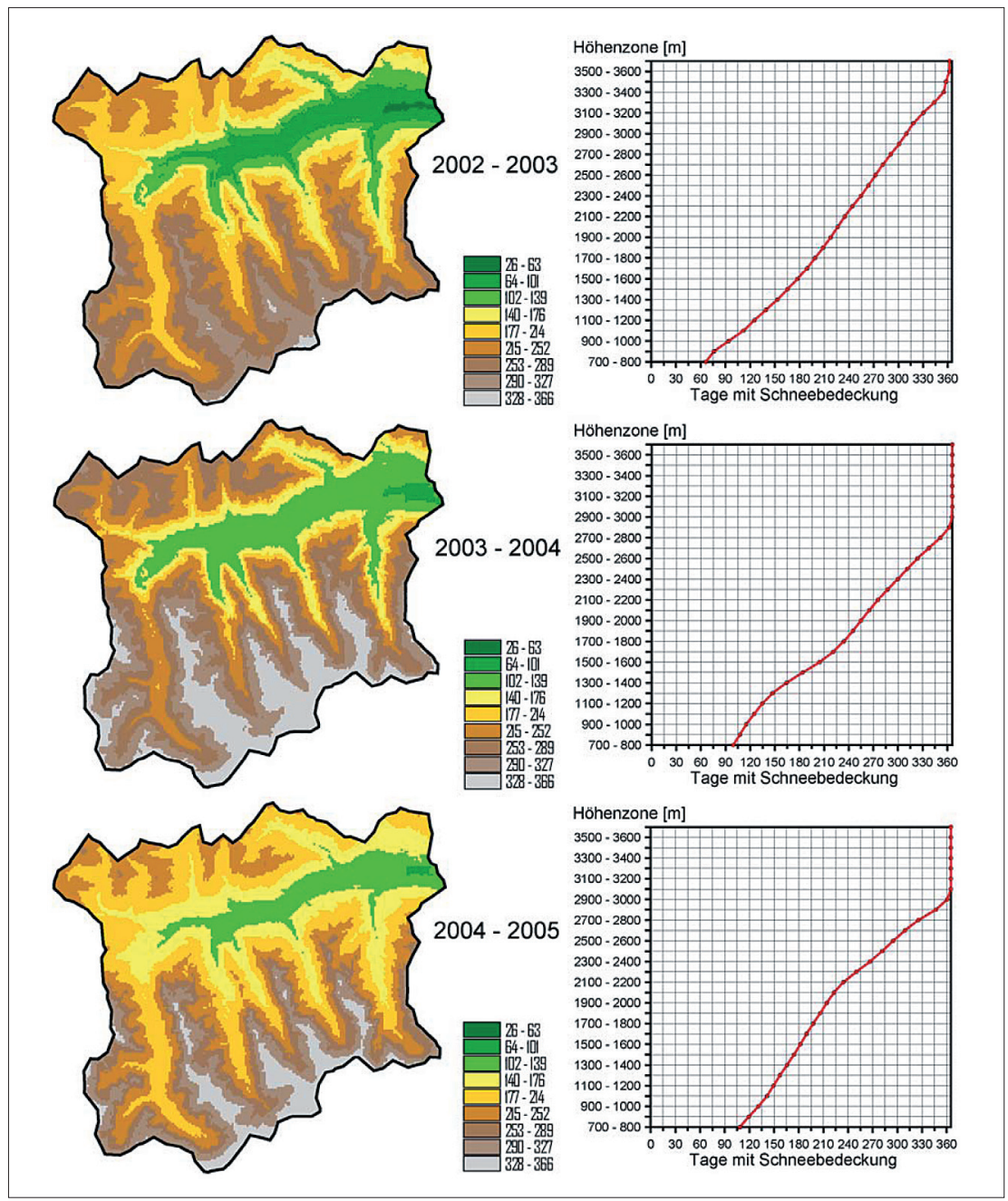

Abb. 5: Räumliche Darstellung der kumulativen Schneedeckendauer der Haushaltsjahre 2002 bis 2005 und mittlere Anzahl der Tage mit Schneebedeckung in den $100 \mathrm{~m}$ - Höhenzonen des Einzugsgebietes Oberer Pinzgau.

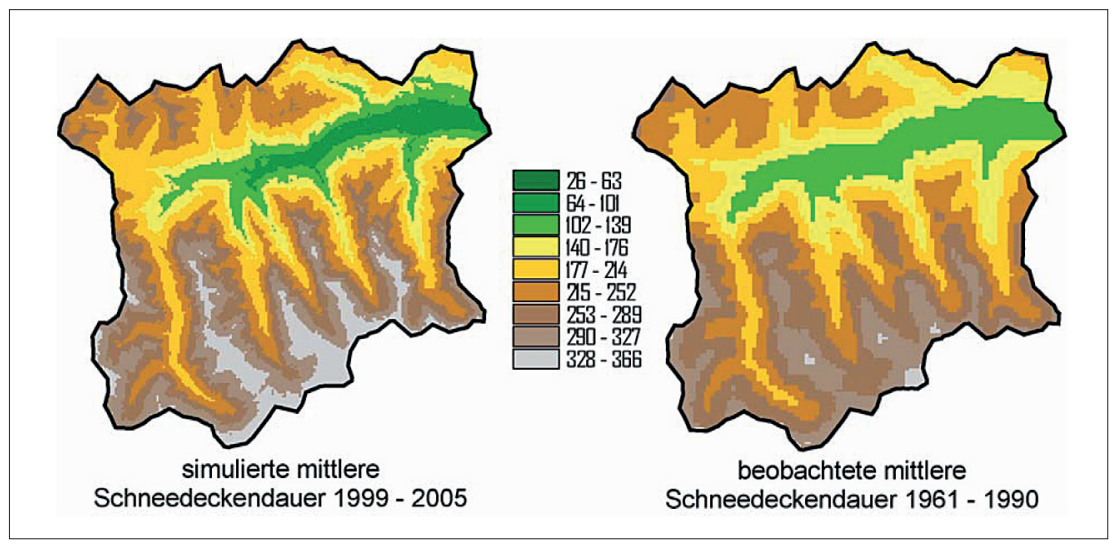

Abb. 6: Räumliche Darstellung der kumulativen Schneedeckendauer der Haushaltsjahre 2002 bis 2005 und mittlere Anzahl der Tage mit Schneebedeckung in den $100 \mathrm{~m}$ - Höhenzonen des Einzugsgebietes Oberer Pinzgau.

Möglichkeit für die Berechnung der kumulativen Schneedeckendauer ergibt sich aus der räum- lichen Interpolation von punktuellen Schneedeckeninformationen (Schöner und Mohnl, 2003). Dabei 
hängt das Ergebnis von der Dichte des Schneedeckenmessnetzes und dem gewählten Interpolationsverfahren $a b$.

Ein großer Nachteil der berechneten Schneedeckendauer aus der hydrologischen Simulation ist die im Modell nicht implementierte Verfrachtung des Schnees durch Wind und Lawinen, welche in der Natur dafür sorgt, dass selbst nach großen Schneefällen hochgelegene
Grate und Gipfel rasch schneefrei werden. Durch den vertikalen Transport gelangt der Schnee in tiefere Lagen und kann dort auch auf Grund der höheren Temperaturen früher abschmelzen. Weiters muss erwähnt werden, dass die aus einem hydrologischen Modell gewonnene Information von mehreren Eingangsvariablen direkt oder indirekt abhängig und damit von Unsicherheiten behaftet ist.
1 Internationale Forschungsgesellschaft INTERPRAEVENT, \% Abt. 18 Wasserwirtschaft, Amt der KärntnenT, \% Abt. 18 Wasserwirtschaft, Amt der 70, 9020 Klagenfurt, Austria

2 Zentralanstalt für Meteorologie und Geodynamik - Klimaabteilung, Hohe Warte 38, 1190 Wien

${ }^{3}$ Universität für Bodenkultur (BOKU) Wien, Institut ür Wasserwirtschaft, Hydrologie und Konstruktiven Wasserbau, Muthgasse 18, 1190 Wien

\section{Korrespondenz:}

Gernot Koboltschnig

Internationale Forschungsgesellschaft INTERPRAEVENT, www.interpraevent.at

E-Mail: gernot.koboltschnig@ktn.gv.at

Tel: +43-463-536-31805, Fax: +43-463-536-31828

\section{LITERATUR}

ASL (2003) Amt der Salzburger Landesregierung Luftbild Orthophotos und digitales Höhenmodell der Hohe Tauern Region

Auer I, Böhm R, Mohnl H, Potzmann R, Schöner W, Skomorowski P (2001) ÖKLIM-Digitaler Klimaatlas Osterreichs. Eine interaktive Reise durch die Vergangenheit, Gegenwart und Zukunft des Klimas (auf CD). Zen

anstalt für Meteorologie und Geodynamik, Vienna. Beniston M (1997) Variations of snow depth and duration in the Swiss Alps over the last 50 years: links to changes in large-scale climate forcings. Climatic Change 36: $281-300$

Brander D, Seidel K, Zurflüh M, Huggel Ch (2000) Snow cover duration maps in Alpine regions from remote sensing data. EARSeL eProceedings 1: 292-296.

Frauenfelder R, Zemp M, Haeberli W, Hoelzle M (2005) Worldwide glacier mass balance measurements: trends and first results of an extraordinary year in Centra Europe. Ice and Climate News 6: 9-10

Gurtz J, Baltensweiler A, Lang H (1999) Spatially distributed hydrotopebased modelling of evapotranspiration and runoff in mountainous basins. Hydrological Processes 13: $2751-2768$

Hantel M, Ehrendorfer M, Haslinger A (2000) Climate sensitivity of snow cover duration in Austria Internation Journal of Climatology 20: 615 640

Hock $R$ (1999) A distributed temperature-index iceand snowmelt mod tion. Journal of Glaciology 45/149: 101-111
Hynek B, Schöner W (2004) Massenhaushalt 2002/2003 der Gletscher in der Goldberggruppe. Jahrbuch des Sonnblick-Vereins, 101-102, 1-15

Kling H, Nachtnebel HP, Fürst J (2005) Bilanzierungsgebiete, klimatische Wasserbilanz und Abflussver. schaft, Unwelt und Wasserwirtschaft (ed.) Hydrologischer Atlas O

Koboltschnig GR, Schöner W, Zappa M, Holzmann H (2008b) Glacier melt of a small basin contributing to runoff under the extreme climate conditions in the summer of 2003. Hydrological Processes. DOI: 10.1002/hyp.7203 Koboltschnig GR, Schöner W, Zappa M, Kroisleitner C, Holzmann H (2008a) Runoff modelling of the glacierized alpine Upper Salzach basin (Austria): Multi-criteri result validation. Hydrological Processes 22: 3950-3964. DOI: 10.1002/hyp.7112

Koboltschnig GR, Schöner W, Zappa M, Holzmann H (2007) Contribution of glacier melt to stream runoff: the climatically extreme summer of 2003 had happened in 1979. Annals of Glaciology 46: 303-308

Nash JE, Sutcliffe JV (1970) River flow forecasting through conceptual models (1), a discussion of principles. Journal of Hydrology 10(3): $282-290$

Parajka J, BlöschI G (2006) Validation of MODIS snow Par ing Sciences 10: 679-689

Randin CF, Engler R, Normand S, Zappa M, Zim- mermann NE, Pearman P, Vittoz P, Thuiller W, Guisan A (2008) Climate change and plant distribution: local models predict high-elevation persistence. Global Change Biology. in print

Schöner W, Mohnl H (2003) Schneehöhen und Schneebedeckung. In: Bundesministerium für Land- und Forstwirtschaft, Umwelt und Wasserwirtschaft (ed.) Hydrologischer Atlas Österreichs, 2. Lieferung, Kartentafe 4.1. Wien; ISBN 3-85437-250-7

Singh P, Spitzbart G, Hübl H, Weinmeister HW (1997) Hydrological response of snowpack under rainon-snow events: a field study. Journal of Hydrology 202 $1-20$

Strasser U, Mauser W (2001) Modelling the Spatial and Temporal Variations of the Water Balance for the Weser Catchment 1965-1994. Journal of Hydrology 254(1-4): 199-214

Viviroli D, Gurtz J, Zappa M (2007) The Hydrological Modelling System PREVAH. Model documentation and user manual. Geographica Bernensia P40. Berne: De partment of Geography, University of Berne, ISBN 9783905835-01-0

Wielke L-M, Haimberger L, Hantel M (2004) Snow cover duration in Switzerland compared to Austria. Meteorologische Zeitschrift 13(1): 13-17

Zappa M, Kan C (2007) Extreme heat and runoff exZappa M, Kan C (200) Extreme hat and runoff exstem Scion 7: 375 389, ww Hazd and Earth System Sciences 7: 375-389, www. nat-hazards-earth-syst- 